\title{
A search for counterparts to massive X-ray binaries using photometric catalogues ${ }^{\star} \star \star$
}

\author{
I. Negueruela ${ }^{1,2}$ and M. P. E. Schurch ${ }^{3}$ \\ 1 Departamento de Física, Ingeniería de Sistemas y Teoría de la Señal, Universidad de Alicante, Apdo. 99, E03080 Alicante, Spain \\ e-mail: ignacio@dfists.ua.es \\ 2 Department of Physics and Astronomy, The Open University, Walton Hall, Milton Keynes MK7 6AA, UK \\ 3 School of Physics and Astronomy, University of Southampton, Southampton SO17 1BJ, UK
}

Received 17 July 2006 / Accepted 25 September 2006

\begin{abstract}
Context. The X-ray and $\gamma$-ray observatory INTEGRAL has discovered large numbers of new hard X-ray sources, many of which are believed to be high mass X-ray binaries. However, for a significant fraction, their counterparts remain unidentified.

Aims. We explore the use of photometric catalogues to find optical counterparts to high mass X-ray binaries and search for objects likely to be early-type stars within the error circles of several INTEGRAL sources.

Methods. Candidates were selected from 2MASS photometry by means of a reddening free $Q$ parameter. Sufficiently bright candidates were spectroscopically observed.

Results. Many of the candidates selected turned out to be moderately reddened late A or early F stars. Optically visible OB stars are very scarce even in these Galactic Plane fields. Our method is able to identify the counterpart to IGR J16207-5129, confirmed by a Chandra localisation. We classify this object as a B0 supergiant. In the field of AX J1820.5-1434, we find a mid or early B-type star, but we cannot confirm it as the counterpart. For IGR J16320-4751 we rule out the optically visible candidate as a possible counterpart. For AX J1700.2-4220, we do not find any suitable candidate within the ASCA error circle. We classify HD 153295, a marginal candidate to be the counterpart, as B0.5 IVe, and find a distance compatible with membership in Sco OB1. In the case of IGR J17091-3624, the object associated with a variable radio source in the field is a late F star. We derive a spectral type B0 IIIe for HD 100199, previously identified as the counterpart to IGR J11305-6256.

Conclusions. The procedure used is able to correctly identify OB stars and, in about one third of the cases, may lead to the localisation of the correct counterpart. However, the majority of INTEGRAL error circles do not contain any suitable optically visible counterpart. Deep infrared searches are going to be necessary in order to locate the counterparts to these sources.
\end{abstract}

Key words. binaries: close - stars: supergiants - X-rays: binaries - stars: emission line, Be

\section{Introduction}

High Mass X-ray Binaries (HMXBs) are X-ray sources powered by accretion on to a compact object of material coming from a companion massive star. HMXBs are objects of the highest astrophysical interest, as their study allows us to address a number of fundamental questions, from the masses of neutron stars to the structure of stellar winds (e.g., Kaper et al. 2004). They can also be used to constrain models of stellar evolution, binary evolution and the mechanisms for the formation of neutron stars and black holes. Moreover, because of their young age, when considered as a population, they can provide information on the properties of galaxies, such as their star formation rates (e.g., Grimm et al. 2003).

During the last couple of years, the ESA X-ray and $\gamma$-ray observatory INTEGRAL has been conducting a continuous monitoring of the Galactic Plane, with especial attention to the

\footnotetext{
* Based on observations collected at the South African Astronomical Observatory.

$\star \star$ Spectra are only available in electronic form at the CDS via anonymous ftp to cdsarc.u-strasbg.fr $(130.79 .128 .5)$ or via http://cdsweb.u-strasbg.fr/cgi-bin/qcat?]/A+A/461/631
}

Galactic Centre and its surroundings. These observations have revealed large numbers of new X-ray sources, many of which appear hard. In most cases, these hard spectra are believed to be due to high interstellar extinction resulting in the absorption of any soft components. A substantial fraction of these sources are believed to be HMXBs, based on their X-ray characteristics (see, e.g., Walter et al. 2006), and in many cases, this has been proved by the discovery of their counterparts (e.g., Filliatre \& Chaty 2004; Reig et al. 2005; Masetti et al. 2006a; Negueruela et al. 2006a).

Interestingly, many of these objects are believed to have OB supergiants as mass donors. In some cases, the X-ray sources are pulsed and orbital parameters typical of persistent Supergiant X-ray Binaries (SGXBs) have been found (e.g., Bodaghee et al. 2006; Zurita Heras et al. 2006). In several other cases, the systems have been identified as supergiant fast X-ray transients (SFXTs), displaying short outbursts (Sguera et al. 2005; Negueruela et al. 2006b; Smith et al. 2006; Sidoli et al. 2006). This is a very important result, because for many years it was thought that the population of SGXBs should be relatively small (because of evolutionary reasons) and that a substantial fraction of its members were already known (because they were 
persistent moderately bright X-ray sources). The discovery of many new systems (currently, INTEGRAL has found or identified more SGXBs than were previously known; cf. Walter et al. 2006) represents a challenge to binary star population synthesis models. Because of this, the search for the counterparts to as many new INTEGRAL sources as possible is a very urgent matter.

Here we report on our search for counterparts to a number of INTEGRAL sources believed to be HMXBs through the selection of candidate OB stars in their error circle based on their 2MASS (Skrutskie et al. 2006) colours. We also present new spectra and spectral classifications for some proposed counterparts.

\section{Methodology and observations}

As is only natural, candidate HMXBs lie close to the Galactic Plane. INTEGRAL error circles have in most cases $2^{\prime}$ radii (some are slightly worse) and therefore contain many dozens of optical and infrared sources. In a few cases, the INTEGRAL source coincides with an ASCA source and then the error circle is smaller $\left(50^{\prime \prime}\right.$ radius), though still relatively large. The number of possible counterparts is simply far too large to tackle.

The situation becomes somewhat better if we expect the counterpart to a given source to be a high mass star. These objects are intrinsically blue and intrinsically bright. Unless they are obscured by enormous amounts of extinction, they are expected to be relatively bright in the $K$ band, where the extinction is $\sim 10$ times lower than in the optical. Because of this, it is not unreasonable to expect to find the counterpart among the stars detected in the 2MASS catalogue.

We have used 2MASS photometric data to search for objects within the X-ray error circles that might be intrinsically blue. Under the assumption of a standard reddening law, which is much more likely to hold in the infrared than in the UV/optical region (e.g., Indebetouw et al. 2005), the observed infrared colours of stars can be projected along a known reddening law to the location of their intrinsic colours. However, in the nearinfrared, the colours of early-type stars are basically degenerate (cf. Ducati et al. 2001, where the $(H-K)_{0}$ colours of B-type and early A-type hardly cover a range of $0.1 \mathrm{mag}$ ) and therefore typical 2MASS errors, of the order of $\sim 0.03-0.05 \mathrm{mag}$ in a given colour even for bright stars, do not allow an accurate dereddening. A simpler approach, such as the calculation of an infrared equivalent to Johnson's $Q$ parameter, is likely to give similar results.

If we define the reddening-free quantity $Q=(J-H)-$ $1.70\left(H-K_{\mathrm{S}}\right)$, the intrinsic colours of early and late-type stars are such that they will lie clearly separated in the $Q / K_{\mathrm{S}}$ diagram (e.g., Comerón \& Pasquali 2005). The majority of stars in Galactic fields concentrate around $Q=0.4-0.5$, corresponding to field $\mathrm{K}$ and $\mathrm{M}$ stars, while early-type stars typically have $Q \simeq 0$.

For early-type supergiants, several factors can affect their intrinsic colours, among them, variations in the extinction law ${ }^{1}$, infrared excesses, etc. Because of this, we preferred to check

\footnotetext{
1 The factor $E(J-H) / E(H-K)=1.70$ is obtained from the extinction law of Rieke \& Lebofsky (1985). The extinction law of Fitzpatrick (1999) gives a value $E(J-H) / E(H-K) \approx 1.9$. As a matter of fact, both extinction laws use Johnson's $K$ band, while 2MASS uses $K_{\mathrm{S}}$. Its shorter wavelength should, in principle, result in higher extinction, though empirical results by Indebetouw et al. (2005) indicate a value $\approx 1.8$. The adoption of $E(J-H) / E\left(H-K_{\mathrm{S}}\right)=1.8$ would only affect our results for the case of heavily reddened stars, resulting in more negative values of $Q$. In view of this, we prefer to stick to the 1.7 value, as the
}

the $Q$ values of known SGXBs, mostly those detected by INTEGRAL, with known counterparts. They have a range of $Q$ values, extending from -0.05 to 0.15 . Therefore we proceeded to search the error circles of INTEGRAL sources selecting sources according to the following criteria:

- we selected stars with $K_{\mathrm{S}}<11$ and $Q<0.2$ as possible OB supergiants (in practice, a value of $Q \lessgtr-0.1$ would indicate an infrared excess, but we did not find any star fulfilling this condition). A magnitude cutoff is reasonable in this case. If a Galactic OB supergiant is fainter than this limit in $K_{\mathrm{S}}$, this would imply amounts of obscuration such that the $J$ band measurement would be unreliable (see typical values in Table 2). Moreover, the object would certainly be too faint in the optical to have been observed for this work;

- we selected stars with $K_{\mathrm{S}}<12$ and $Q<0$ as possible Be stars. Be stars are characterised by strong infrared excesses and application of a dereddening procedure that assumes standard reddening results in an "over-correction" that leads to negative $Q$ values. Obviously, we could have found Be stars at magnitudes fainter than $K_{\mathrm{S}}=12$, but again those objects would be too reddened to have reliable $J$ magnitudes; - we rejected stars whose USNO colours were incompatible with a reddened $\mathrm{OB}$ star, i.e., objects that were too bright in the blue band compared to their $J$ magnitude and $(J-K)$ colours. As a reference, the standard reddening law implies $E(B-R)=3.4 E(J-K)$ and so an OB star with $(J-K) \approx 1$ should have $(B-R) \gtrsim 3$.

We tested this method on the fields of five SGXBs detected by INTEGRAL with known counterparts, all of which are relatively faint in the optical. In all cases, the counterpart was selected among the very few candidates complying with the criteria. Application of this method has led to the identification of an obscured OB supergiant in the field of the SFXT SAX J1818.6-1703 (Negueruela \& Smith 2006). However, the criteria do not guarantee the selection of an OB star. Reddened A-type and early F-type stars will still have $Q<0.2$ (A stars will actually be quite close to $Q=0$ ), while oxygen-rich AGB stars and carbon-rich giants can display $J H K_{\mathrm{S}}$ colours indistinguishable from those of normal, reddened early-type stars (Bessell \& Brett 1988; Comerón \& Pasquali 2005). Moreover, all kinds of stars with infrared excess, such as T Tauri stars, will have $Q<0$. Spectroscopic observations are thus required to confirm the nature of the candidates.

Observations of the candidate stars were carried out on the nights of 2006 May 2nd to 8th using the unit spectrograph on the 1.9-m telescope at the South African Astronomical Observatory in Sutherland ${ }^{2}$. We used gratings \#8 and \#9 to observe objects in the red region and grating \#6 to observe them in the blue. In all cases, we used a 1'.5 slit.

Grating \#8 (830 $\mathrm{ln} / \mathrm{mm})$ was used on the nights of May 4th and 6th. It covers the $6200-7400 \AA$ range with a resolution element (measured on arc frames) of $1.5 \AA$ around $\lambda 7000 \AA$.

Grating \#9 was used on the night of May $7 \mathrm{th}$. It covers the 6200-8900 $\AA$ region with a resolution element (measured on arc frames) of $3.2 \AA$ around $\lambda 7500 \AA$.

location of stars in this diagram is well explored and the $Q$ values of $\mathrm{OB}$ stars are known to be well separated from those of later-type field stars.

${ }^{2}$ http://www.saao.ac.za/facilities/instrumentation/ gratingspec/ 


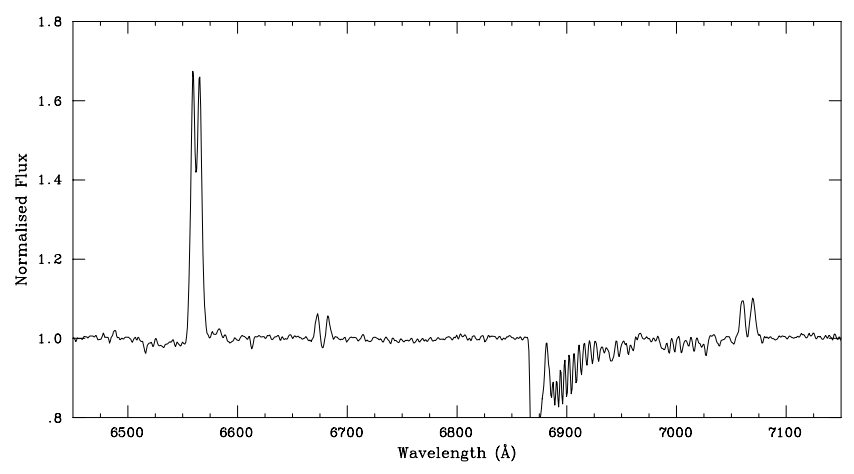

Fig. 1. Red spectrum of HD 100199, the optical counterpart to IGR J11305-6256, showing the clearly separated double peaks in all emission lines. The central absorption in He I $6678 \AA$ comes below the continuum level, indicating that HD 100199 is a shell star.

Grating \#6 (600 ln/mm) was used on the night of May 8th. It covers the 3800-5600 $\AA$ range with a resolution element (measured on arc frames) of $2.3 \AA$ around $\lambda 4500 \AA$.

Two faint targets were later observed on the night of June 5th 2006 with the 2.6-m Nordic Optical Telescope (NOT), in La Palma (Spain), equipped with the Andalucia Faint Object Spectrograph and Camera (ALFOSC) ${ }^{3}$. We used grism \#4, which provides a dispersion of $3 \AA$ /pixel over the whole optical range.

All the spectra have been reduced with the Starlink packages CCDPACK (Draper et al. 2000) and FIGARO (Shortridge et al. 1997) and analysed using FIGARO and DIPSO (Howarth et al. 1998).

\section{Results}

\subsection{IGR J11305-6256}

The weak transient source IGR J11305-6256 was detected by IBIS/ISGRI in May 2004 (Produit et al. 2004). Masetti et al. (2006a) noted that the catalogued Be star HD 100199 was within the large ( $5^{\prime}$ radius) error circle, and later confirmed the identification with a $S W I F T / X R T$ detection (error circle of radius only 6"). Quoting a spectral type of B0 IIIe from Garrison et al. (1977), they estimate a distance of $\sim 3 \mathrm{kpc}$. We note that, with $Q=-0.12$, HD 100199 is the only star standing out in the $Q / K$ diagram for the error circle, though there are a couple other marginal candidates.

As the only modern spectrogram of this source is the low resolution spectrum of Masetti et al. (2006a), we have obtained intermediate resolution spectra of HD 100199, in order to reassess its spectral classification. The red spectrum of HD 100199 (see Fig. 1) displays double-peaked emission lines corresponding to $\mathrm{H} \alpha$ and He I 6678, $7065 \AA$. Masetti et al. (2006a) report singlepeaked emission in $\mathrm{H} \alpha$, but this is likely to be due simply to the low resolution of their spectrum. There is a weak emission line around $\lambda 6582 \AA$, which may be due to the C II $6578,6582 \AA$ doublet.

The blue spectrum (see Fig. 2) shows double-peaked emission in $\mathrm{H} \beta$ and emission infilling in $\mathrm{H} \gamma$. Four He II lines are visible. In particular, the prominent He II $5412 \AA$ line (not shown) and the presence of a weak He II $4542 \AA$ indicate that the star

\footnotetext{
${ }^{3}$ http://www.not.iac.es/instruments/alfosc/
}

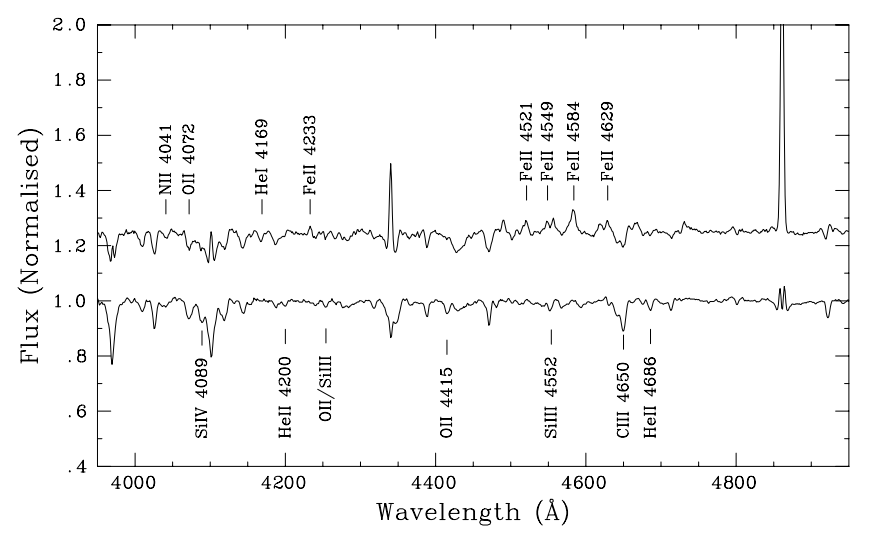

Fig. 2. Classification spectra of HD 100199 (bottom), the optical counterpart to IGR J11305-6256, and HD 153295 (top), possible counterpart to AX J1700.2-4220.

has to be classified B0 or earlier. The fact that He II $4542 \AA<$ Si III $4552 \AA$ prevents an O-type classification and sets the spectral type at B0. The weakness of He II $4686 \AA$, which is inversely correlated with luminosity class, shows that this star is at least of moderate luminosity. The intensity of the C III $4650 \AA$ line when compared to He I lines would suggest a very high luminosity, as it is clearly stronger than He I $4471 \AA$. However, this high luminosity is not supported by the ratio of Si IV lines to He I lines or the fact that He I $4713 \approx \mathrm{He}$ II $4686 \AA$. Therefore we adopt a luminosity class III, confirming the classification of Garrison et al. (1977), though noting the abnormal strength of C III $4650 \AA$.

As the $\mathrm{H} \alpha$ emission is not strong $(E W=-7 \AA)$, the contribution of the circumstellar disk to $E(B-V)$ will likely not be very large, and so the distance calculated by Masetti et al. (2006a) is a good estimate. This object is likely to be a persistent lowluminosity Be/X-ray binary.

\subsection{IGR J16207-5129}

This source appears in the first INTEGRAL catalogue (Bird et al. 2004) as INTEGRAL1 22. Tomsick et al. (2006) selected it as a seemingly persistent source, likely to be a HMXB, and observed it with Chandra, resulting in an accurate localisation. Masetti et al. (2006b) have obtained a spectrum of the only object in the Chandra error circle and reported it to be a reddened emission-line star.

This source offers a suitable example for the application of the $Q$ method. Figure 3 shows a plot of $Q$ against $K_{\mathrm{S}}$ magnitude for all stars brighter than $K_{\mathrm{S}}=14$ within a $3^{\prime}$ radius from the nominal position of IGR J16207-5129. The four stars fulfilling our criteria stand clearly on the left top corner of the plot. However, the magnitudes of the object with $Q=-0.30$, 2MASS J16202935-5130457 are flagged as bad. Therefore its $Q$ value is likely to be meaningless, and we do not consider it a real candidate to be a Be star. The bright source 2MASS J16203633-5129352 corresponds to the catalogued emission-line star HD 146803, reported by Masetti et al. (2006a) to be a late Be star and so not a likely candidate. This leaves us with two viable candidates. 2MASS J16202603-5129275 has colours and magnitudes typical of an early-type supergiant. However, close examination of the images available suggests that the optical source USNO-B1.0 0385-0554515 is not the same object, as its position is displaced by $\sim 2^{\prime \prime}$ and DENIS identifies two separate objects here, one coincident with the 


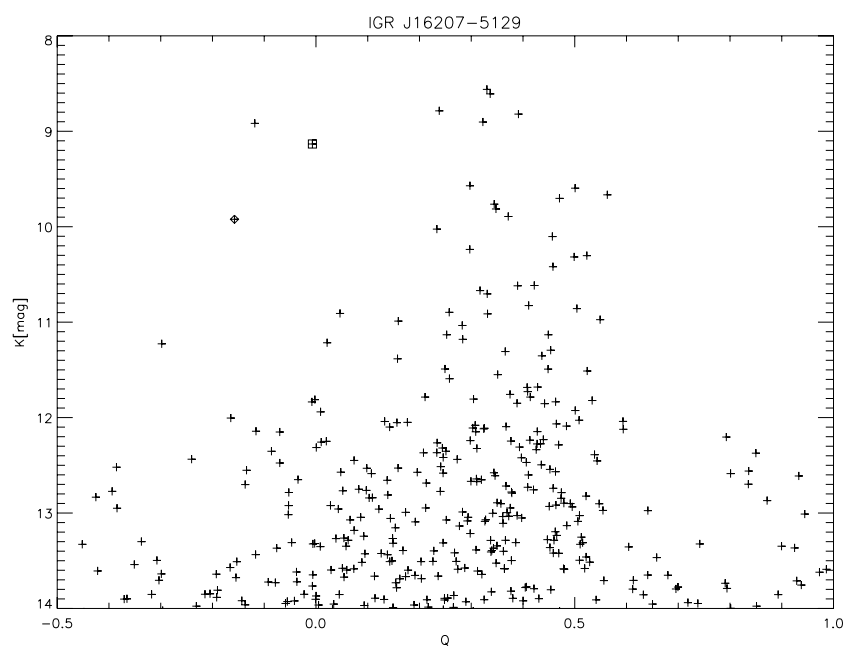

Fig. 3. A plot of IR $Q$ values against $K_{\mathrm{S}}$ magnitude for 2 MASS stars within $3^{\prime}$ of the position for IGR J16207-5129. Candidate early-type stars clearly stand out on the top left corner. The object marked with a diamond is J16203633-5129352 = HD 146803, while the object marked with a square is J16204627-5130060, the correct counterpart. The brightest candidate, J16202603-5129275, seems to be resolvable into two objects. Typically, errors in $K$ run from $\sim 0.02$ for the brightest objects to $\sim 0.04$ at $K_{\mathrm{S}}=14$, but can be rather larger for blended objects (we did not select candidates with $\Delta K>0.05$ ). Errors in $Q$ vary from $\Delta Q=0.04$ to rather high values, with a mean value of $\Delta Q=0.11$ and a median $\Delta Q=0.10$.

2MASS source and one coincident with the USNO source. This means that the $J$ mag is likely to represent a blend of both objects and so, again, the $Q$ value is meaningless.

The only candidate left, 2MASS J16204627-5130060 = USNO-B1.0 0384-0560875, was confirmed as the counterpart to IGR J16207-5129 by a Chandra localisation shortly before our run (Tomsick et al. 2006; Masetti et al. 2006b). USNO-B1.0 quotes $B=19.7$ for this object, far too faint for a classification spectrum. However, the $I$-band spectrum can be used to classify luminous $\mathrm{OB}$ stars following the prescriptions of Caron et al. (2003) and Clark et al. (2005).

We observed USNO-B1.0 0384-0560875 in the red and far red. Its spectrum is shown in Fig. 4. The spectrum shows deep and well separated Paschen lines, several He I lines and no obvious metallic lines. The Paschen lines are well separated and resolvable up to at least $\mathrm{Pa} 20$. The $\mathrm{He}$ I lines are resolved even at this low resolution. These features clearly identify the object as an OB supergiant. The lack of O I $8446 \AA$ and other metallic lines makes it earlier than B3. Moreover, the lack of O I $7774 \AA$ indicates that it is earlier than B1. A limit on the hot side is more difficult to set. However, He II $6683 \AA$ A becomes clearly resolved from He I $6678 \AA$ around O8.5 and there is no sign of it in our spectrum. Moreover, the Paschen lines tend to become shallower and less well defined in O-type supergiants. Therefore we conclude that this object has a spectral type of B0 with an uncertainty of about one subtype.

In view of its optical counterpart and persistent nature, IGR J16207-5129 is a new SGXB.

\subsection{IGR J16283-4843}

This source has been observed by different X-ray observatories (Beckmann et al. 2005). It presents a hard spectrum, with very high intrinsic absorption. The X-ray spectrum strongly
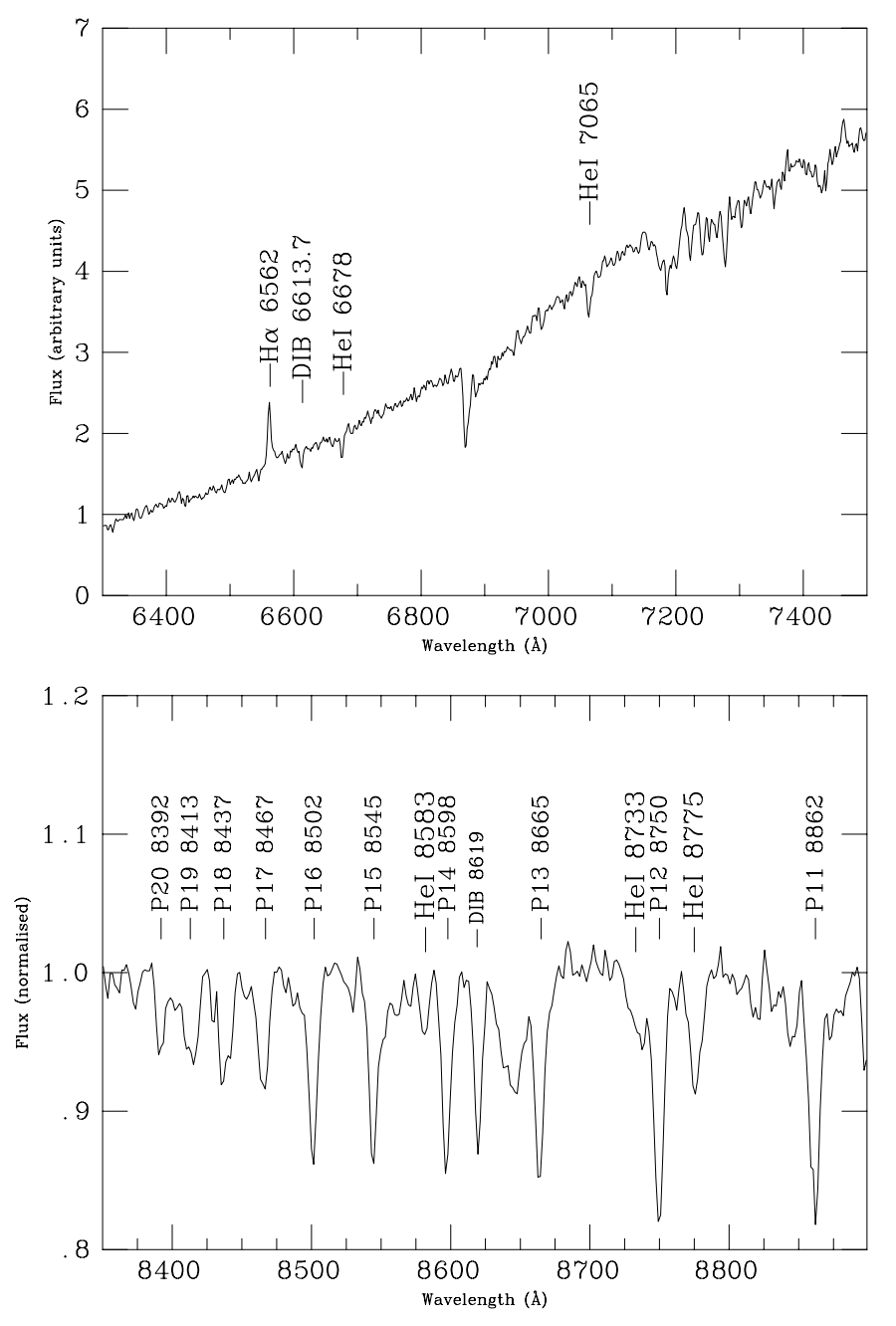

Fig. 4. Spectrum of USNO-B1.0 0384-0560875, the optical counterpart to IGR J16207-5129. The top panel shows the red part of the spectrum, while the lower panel zooms in the region around the Paschen lines. The features in this region clearly identify the star as an early B supergiant.

suggests that it is a HMXB (Beckmann et al. 2005). The SWIFT error circle encompasses a single 2MASS source, 2MASS J16281083-4838560. This object is in reality a blend of images of at least three stars (Steeghs et al. 2005) that coincides with a Spitzer source (Beckmann et al. 2005). The mid-IR detection favours the identification of this source as the counterpart, but its faintness $(K=13.95)$ is surprising for the counterpart of a HMXB.

Because of this, we searched the vicinity of this position and found a bright 2 MASS source, $\approx 9^{\prime \prime}$ away from the centre of the error circle, 2MASS J16280983-4838590. This object is relatively bright $K=12.01$ and has $Q=-0.3$, fulfilling our conditions for a candidate Be star. However, deep $K$-band images of this area by Steeghs et al. (2005) show that there are several fainter $K$-band sources surrounding 2MASS J16280983-4838590 and suggest that this source is actually a blend of two objects of similar magnitudes.

A coincident optical source, USNO-B1.0 0413-0504855 $\left(R_{1}=14.2\right)$ could correspond to 2MASS J16280983-4838590, but it looks far too bright in the optical to be the same as an object with $\left(J-K_{\mathrm{S}}\right)=1.3$. In any event, we observed it and it turns out to be a K-type star. Obviously, a deep IR investigation of this error circle is necessary. 
Table 1. Candidate blue stars in the field of AX J1700.2-4220. Note that the third candidate is formally outside the ASCA error circle.

\begin{tabular}{ccccc}
\hline \hline 2MASS & USNO-B1.0 & $Q$ & $K$ & $R_{2}$ \\
\hline J17001950-4219410 & $0476-0587824$ & -0.15 & 11.65 & 13.7 \\
J17001531-4219395 & $0476-0587760$ & -0.08 & 11.30 & 14.3 \\
J17001663-4219078 & $0476-0587779$ & +0.02 & 10.58 & 13.4 \\
\hline
\end{tabular}

\subsection{IGR J16320-4751}

This INTEGRAL source, also known as AX J1631.9-4752, is a $P_{\mathrm{s}}=1300 \mathrm{~s}$ pulsar (Rodriguez et al. 2006; Lutovinov et al. 2005a). Monitoring with the SWIFT/BAT instrument has resulted in the discovery of an 8.96-d periodicity in its light curve (Corbet et al. 2005). The X-ray spectrum of IGR J16320-4751 suggests it is a HMXB, while the periodicities detected are typical of SGXBs. There is one source inside the XMM-Newton error circle, 2MASS J16320175-4752289, and a second one just outside. 2MASS J16320175-4752289, is rather faint. 2MASS gives $K=10.99, H=13.03$ and only an upper limit in $J$. Therefore we could not calculate its $Q$ value. The other source, 2MASS J16320215-4752322 is visible in the optical as USNO-B1.0 0421-0625270 (with $R_{2}=15.4$ ). This object, with $K=10.8$ and $Q=0.18$, is marginally consistent with being a reddened $\mathrm{OB}$ supergiant. However, our spectrum shows that 2MASS J16320215-4752322 is a K-type giant. This suggests that 2MASS J16320175-4752289 is the actual counterpart to this HMXB, as seems to be confirmed by the report by Walter et al. (2006) that it is an early-type star. If it is an OB supergiant, it has to be extremely reddened.

\section{5. $A X J 1700.2-4220$}

An INTEGRAL source coincident with this faint ASCA source is listed in the 1st INTEGRAL catalogue (Bird et al. 2004). Masetti et al. (2006b) mention that the catalogued Be star HD 153295 is within the INTEGRAL error circle. However, it is outside the smaller ASCA error circle. Hence we looked for other candidates within the ASCA error box. Analysis of the 2MASS stars within the error circle gives two good candidates to be emission-line early-type stars within the ASCA source and one further candidate to be a supergiant just outside it (see Table 1).

The low resolution spectrum of 2MASS J170015314219395 shows both a strong Ca II triplet and deep, well defined Paschen lines, suggesting that this is a late A/early F star of at least moderate luminosity. We obtained a blue spectrum, which, though rather noisy, suggests that the spectral type is not far from F2 III. 2MASS J17001950-4219410 shows a broad H $\alpha$ absorption line and some weak metallic lines, indicative of a late A-type star, likely of moderate luminosity. Though both stars are relatively early, none is a believable candidate. From the richness of metals in its spectrum and the strength of the metallic blend around $6495 \AA$, 2MASS J17001663-4219078 is a G-type star. The reddening in this direction is perhaps anomalous, as all three stars appear to show $Q$ parameters that are much more negative than the values that correspond to their spectral types.

The lack of convincing candidates within the ASCA error circle opens up the possibility that HD 153295 is the actual counterpart. As expected for a bright Be star, HD 153295 fulfills our conditions with $K=6.73$ and $Q=-0.26$. We obtained intermediate resolution spectra of this star. The red spectrum shows several weak metallic lines and a prominent unresolved $\mathrm{H} \alpha$ emission line with $E W=-68 \pm 2 \AA$. This is quite a high value for a Be star. The blue spectrum (see Fig. 2) confirms the strong Be characteristics. The spectral type is difficult to assign because of the presence of many weak emission lines, but the observed absorption lines place it not very far away of B0.5 IVe. Garrison et al. (1977) give B1? III, in rather good agreement. There are several $U B V$ photometric measurements for this star, showing very little variability. Taking, for example, Schild et al. (1971), we have $V=9.04,(B-V)=0.55$. The strong Be characteristics of HD 153295 imply an important contribution of the circumstellar disk to both $E(B-V)$ and $V$, likely on the order of $E(B-V)_{\text {disk }} \approx 0.15$ and $\Delta V_{\text {disk }} \approx 0.5$ (cf. Dachs et al. 1988).

Assuming standard colours and magnitudes for HD 153295, $(B-V)_{0}=-0.24$ (Wegner 1994) and $M_{V}=-4.5$ (Humphreys $\&$ McElroy 1984), and a standard reddening law, we derive $d \approx 1.7 \mathrm{kpc}$ (a similar value is obtained from the 2MASS magnitudes). This is actually a lower limit to the distance. If we assume the likely contribution from the circumstellar disk given above, we obtain $d \approx 2.6 \mathrm{kpc}$ This distance is comparable to estimates around $2 \mathrm{kpc}$ for the nearby cluster NGC 6231 (e.g., Raboud et al. 1997, and references therein), believed to be the core of the Sco OB1 association. If we were to adopt a luminosity class V for HD 153295, the distance would be fully compatible with membership in Sco OB1.

If HD 153295 is indeed an outlying member of Sco OB1, it may be too young to be a Be/X-ray binary. NGC 6231 is estimated to be very young ( 4 Myr), while typical evolutionary timescales to form Be/X-ray binaries are $\sim 10$ Myr. Thé et al. (1994) have discussed the possible association of HD 153295 with the IRAS source 16569-4213. If the association is true, HD 153295 would be a Herbig Be star and certainly not an X-ray binary, but this would imply an age much younger than $\sim 4 \mathrm{Myr}$ (contraction times for a B0 star are $\sim 10^{5} \mathrm{yr}$; cf. Palla \& Stahler $1999)^{4}$. Clearly, a better position for AX J1700.2-4220 is necessary before the association with HD 153295 can be considered with any confidence.

\subsection{IGR J17091-3624}

First seen by INTEGRAL in April 2003 (Kuulkers et al. 2003), IGR J17091-3624 was later found in archival observations taken by several other satellites (Capitanio et al. 2006). The source is very variable in flux and spectral index, and shows little absorption. Its spectrum can be very hard or relatively soft, suggesting it is a black hole (Lutovinov et al. 2005b; Capitanio et al. 2006). Rupen et al. (2003) found a variable radio source within the error circle with the VLA. Though these findings suggest that IGR J17091-3624 is a low-mass microquasar, we were intrigued to find that the only candidate blue star in the error circle, 2MASS J17090199-3623260, was within the VLA source error circle.

Later, Pandey et al. (2006) observed a radio source at a position coincident with that reported by Rupen et al. (2003). According to Pandey et al. (2006), the only possible counterpart to these sources is 2MASS J17090199-3623260. This object is also detected in the optical as USNO-B1.0 0536-0466988. It has $K=11.7, Q=0.0$ and $R_{1}=14.46$ It should be noted that both the $J$ and $K$ magnitudes for 2MASS J17090199-3623260 are tagged as upper limits. However, they are fully compatible within the errors with the corresponding DENIS magnitudes.

The spectrum of USNO-B1.0 0536-0466988 is displayed in Fig. 5. The $I$-band spectrum shows prominent narrow

\footnotetext{
4 Also, there is another emission line star in the area that could be associated with the IRAS source, GSC 07877-00412.
} 


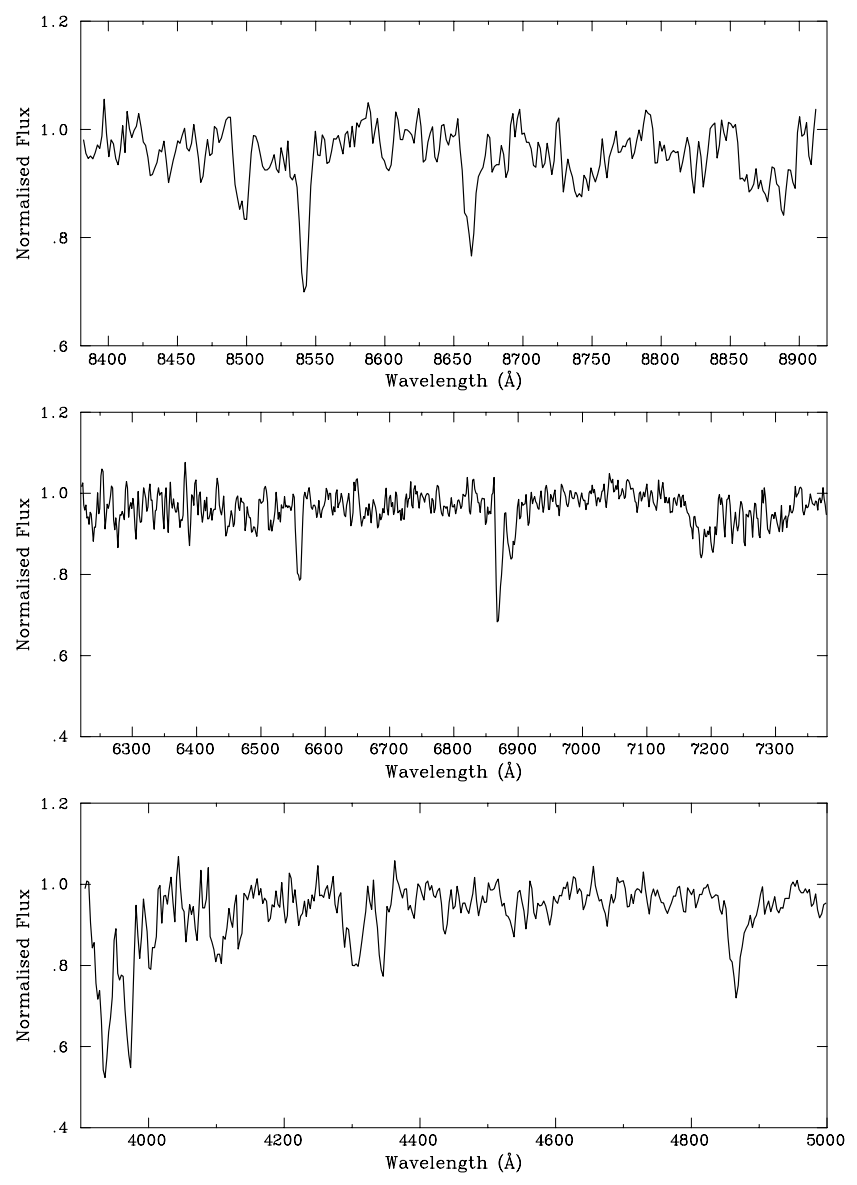

Fig. 5. Several regions of the spectrum of USNO-B1.0 0536-0466988, likely associated with IGR J17091-3624. While the blue and I-band spectra indicate a late $\mathrm{F}$ type, the red spectrum looks more typical of a rather later spectral type, late $\mathrm{G}$ or early $\mathrm{K}$.

Ca II triplet lines and weak Paschen lines, suggesting a mid or late F-type star of moderate luminosity. The low resolution spectrum taken with the NOT covers the blue range. Based on the features present, we estimate a spectral type around F8 V. However, the spectrum around $\mathrm{H} \alpha$ resembles a rather later star. In any case, this object is not an early type star and does not show any sign of emission lines.

In view of the almost secure association with the radio source, we may speculate that IGR 17091-3264 could be a lowmass transient microquasar observed in quiescence. According to Capitanio et al. (2006), the source has not been active since 2004. Perhaps the $J H K$ observations were made at a time when the source was active and the colours dominated by an accretion disk, mimicking the flat IR spectrum of an early type star. We must note, however, that there there is a second faint 2MASS source partially blended with our target, 2MASS J17090219-3623292, which has $H=13.84$ and uncertain $J$ and $K$ magnitudes.

If the 2MASS magnitudes are intrinsic to the star, we can estimate its distance, assuming $M_{V}=+3.5$ for an F8 V star (Mikami \& Heck 1982) and $(V-K)_{0}=1.12$ (Koornneef $1985)$. By using $E\left(J-K_{\mathrm{S}}\right)=1.3 A_{K_{\mathrm{S}}}$ (assuming the extinction law of Rieke \& Lebofsky 1985), we obtain $A_{K_{\mathrm{S}}} \approx 0.2$ and hence $d \sim 800 \mathrm{pc}$. This is certainly much closer than the Galactic Bulge, where most low mass X-ray binaries are known to reside and this source was suspected to lie (e.g. Capitanio et al. 2006). Even if USNO-B1.0 0536-0466988 is the actual counterpart, and the 2MASS magnitudes were obtained during a bright state and they come from an accretion disk, the source is very unlikely to be as far as the Galactic Bulge.

\section{7. $A X J 1820.5-1434$}

This source was discovered by ASCA in 1997 (Kinugasa et al. $1998)$, as a moderately faint X-ray pulsar $\left(P_{\mathrm{s}}=152.3 \mathrm{~s}\right)$. Its $\mathrm{X}$ ray spectrum is highly absorbed, while its transient nature suggests a Be/X-ray binary. INTEGRAL has detected this source up to $70 \mathrm{keV}$ (Filippova et al. 2005).

Analysis of the 2MASS data for the error circle reveals a single candidate to being an early type star, 2MASS J18203114-1434193. This object has $K=10.98$ and $Q=0.06$. The corresponding optical source is USNO-B1.0 0754-0489829, with $R_{2}=14.7$ and $B_{2}=16.5$.

Our spectrum of this source has a very low signal to noise ratio. $\mathrm{H} \alpha$ is seen as a broad absorption feature (with perhaps some emission infilling, though this could be due to the noise) and there are no indications of metallic lines. 2MASS J18203114-1434193 is hence an early type star, but its faintness prevented us from taking a blue spectrum from SAAO. A low resolution spectrum was taken later from the NOT. The spectrum is of moderate signal to noise and low resolution, but the star is certainly a B-type star. The object is not a late B star, as several He I lines are clearly present, but a better spectrum will be necessary to determine an accurate spectral type.

In the NOT spectrum, $\mathrm{H} \alpha$ is seen in absorption. However, many Be/X-ray binaries are known to spend part of the time in absorption-line phases. Therefore, if 2MASS J18203114-1434193 is an early B-type star, it should be considered as a possible counterpart for AX J1820.5-1434.

\subsection{IGR J18406-0539}

The existence of IGR J18406-0539 is dubious. It is located only 3'.5 from the SFXT AX J1841.0-0535 = IGR J18410-0535. So, though formally the error circles for both sources do not overlap (AX J1841.0-0535 has a Chandra localisation), there is a nonnegligible chance that they are the same source (Masetti et al. 2006c). This is a very rich field, but Masetti et al. (2006c) note the presence of the catalogued emission-line star SS 406 on the edge of the error circle. From a low resolution spectrum, they deduce that it is a mid Be star, which, they argue, could be associated with IGR J18406-0539.

The observed spectrum of SS 406 (see Fig. 6) is strange. The $\mathrm{H} \alpha$ spectrum, obtained on the night of May 5th, displays a moderately strong $\mathrm{H} \alpha$ emission line, shallow He I 6678 and $7065 \AA$ lines and some sharp metallic lines. The blue spectrum, taken 3 nights later, displays very narrow Balmer lines, weak sharp He I lines and a wealth of metallic lines. The observed metallic lines do not match a typical F-G spectrum, but rather suggest that SS 406 is a chemically peculiar B-type star. Comparison of the observed metallic spectrum with those of several CP stars suggests that SS 406 is a Mn-Hg star, while the narrowness of its Balmer lines would point to a moderate luminosity.

Chemically peculiar stars are known to be slow rotators, while Be stars are fast rotators. Moreover, the moderately strong He I lines seen in the red spectrum seem incompatible with a late-B CP star. We can think of two explanations for this combination of spectral features. Perhaps SS 406 is a binary composed of a mid Be star and a late-B CP giant. Alternatively, a CP star may show a rotationally modulated spectrum, with 


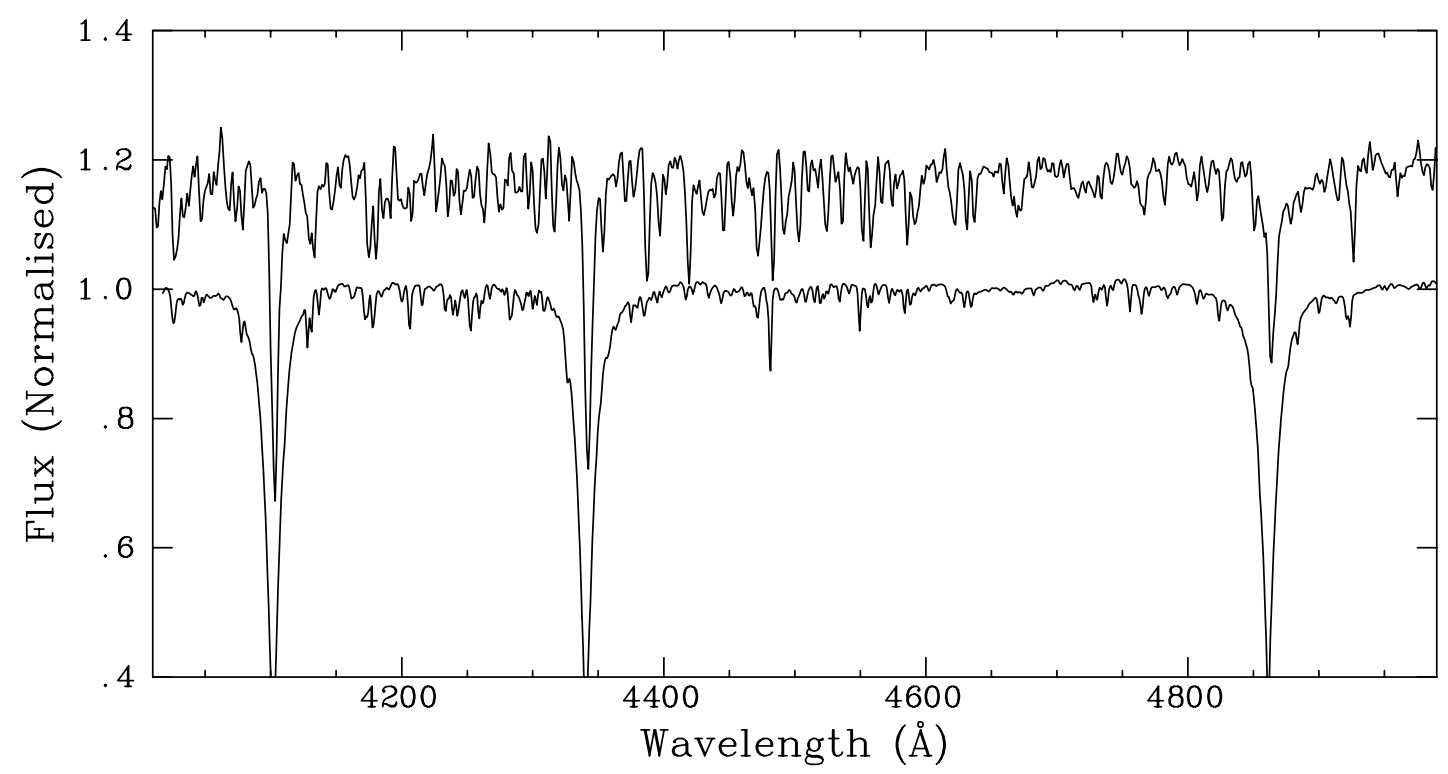

Fig. 6. The blue spectrum of SS 406 (top) compared to the Mn-Hg B8p star HD 221507 (a binned version of a UVES spectrum from the UVES Paranal Observatory Project; Bagnulo et al. 2003). SS 406 has much narrower Balmer lines, but the species present in both spectra mostly match. The presence of stronger He I lines suggests that SS 406 is slightly earlier.

He I and metallic lines changing in appearence and intensity. Whatever the case, SS 406 does not look like a promising counterpart to IGR J18406-0539, if this is indeed a real source. Even if there is a Be star in the system, it is unlikely to be sufficiently early to fall in the range observed for Be/X-ray binaries. Moreover, the evolutionary history of such a system is difficult to conceive.

The $Q$ method reveals a wealth of possible early-type counterparts within the error circle of IGR J18406-0539, though many of them have magnitudes flagged as bad. A correct identification of this source must wait for a better localisation. We note that no X-ray source is visible in this area in the Chandra pointing that led to the localisation of AX J1841.0-0535.

\subsection{Other fields}

Our method did not find any suitable candidate in the field of the SFXT IGR J16479-4514. We did not find any reliable candidates in the field of the SGXB IGR J16418-4532. In this field, few 2MASS stars appear unblended. We observed two very marginal candidates (both with $Q=0.15-0.20$ ) whose 2MASS magnitudes were flagged as bad and both turned out to be late F-type stars. Finally, no candidate blue stars are found in the field of the hard transient IGR J11321-5311.

\section{Conclusions}

1. We have used existing photometric catalogues to search for early type stars within the error circles of X-ray sources believed to be high mass X-ray binaries. Our main discriminant has been the infrared $Q$ parameter, while optical magnitudes from USNO B1.0 have been used in an attempt to rule out foreground A or F-type stars. The method has proved efficient at finding reddened OB stars, resulting in the detection of the likely counterpart to SAX J1818.6-1703 (Negueruela \& Smith 2006) and the selection of the counterpart to IGR J16207-5129, later identified through a Chandra pointing. However, it does not allow the discrimination of reddened A or F-type stars. We have found that many bright candidates selected with $Q \sim 0.1$ turn out to be reddened A or F giants. Most sources with $Q=0.15-0.20$ are reddened late F or G stars. Surprisingly, in a few cases, the candidates turn out to be late-type stars. The most likely explanation for these misidentifications is that the 2MASS sources must be unresolved blends and therefore the $Q$ values calculated lack astrophysical meaning.

2. Recently, Walter et al. (2006) have reported on XMM-Newton localisations of 10 INTEGRAL sources, nine of which they believe to be SGXBs. It is worth noting that, from those 9 sources, the three objects with confirmed early-type luminous counterparts, IGR J16318-4848, IGR J16465-4507 and IGR J17544-2619, would have been selected as candidate OB supergiants by our method, but the proposed candidates for IGR J16393-4641, IGR J16418-4532, IGR J16479-4514 and IGR J172523616 would not (see Table 2). The proposed candidates for IGR J16320-4751 and IGR J18027-2016 lack reliable $J$ magnitudes and so $Q$ values could not be calculated.

While, for example, the proposed counterpart to IGR J16479-4514 fails the $Q$ value criterion (perhaps indicating contamination of the 2MASS magnitudes by other nearby sources), the proposed counterparts to several sources fail the magnitude limit. In the cases of IGR J16283-4843 and IGR J16320-4751, the proposed counterparts are very faint in $K$. Taking into account the huge intrinsic luminosity of an $\mathrm{OB}$ supergiant, these counterparts must be very heavily absorbed not only in the optical, but also in the infrared. If this absorption was mostly intrinsic to the sources (i.e., a very thick wind or dusty envelope surrounding the system), we would expect the systems to be bright mid-IR sources. This is certainly the case in IGR J16318-4848 (Filliatre \& Chaty 2004) and could also happen for IGR J16283-4843, perhaps associated with a mid IR source. However, in other cases, like IGR J16320-4751, there is no such mid IR emission and it seems very unlikely that the absorption occurs in the system itself. The possibility that these objects are HMXBs with main-sequence primaries should be considered. (cf. Pfahl et al. 2002; Ribó et al. 2006). 
Table 2. Summary of results. The top panel contains data for the proposed counterparts to five sources detected with INTEGRAL which had been identified before this work. References are Filliatre \& Chaty (2004), Negueruela et al. (2005), Negueruela et al. (2006a), Pellizza et al. (2006) and Negueruela \& Smith (2006) respectively. The middle panel summarises the results presented here. The bottom panel presents data for the counterparts to four other INTEGRAL sources proposed as HMXBs by Walter et al. (2006).

\begin{tabular}{|c|c|c|c|c|c|c|c|c|c|}
\hline X-ray source & $\begin{array}{c}\text { 2MASS } \\
\text { source }\end{array}$ & $\begin{array}{c}\text { USNO B1.0 } \\
\text { source }\end{array}$ & $\overline{\bar{Q}}$ & $\overline{\overline{K_{\mathrm{S}}}}$ & $\overline{\bar{B}}$ & $\overline{R_{1}}$ & $\begin{array}{c}\text { Counterpart } \\
\text { correct }\end{array}$ & $\begin{array}{l}\text { Spectral } \\
\text { type }\end{array}$ & $\overline{\text { Comments }}$ \\
\hline IGR J16318-4848 & J16314831-4849005 & & -0.11 & 7.19 & & & yes & $\mathrm{B}[\mathrm{e}]$ & obscured \\
\hline IGR J16465-4507 & $\mathrm{J} 16463526-4507045$ & $0448-0520455$ & +0.06 & 9.84 & $15.2\left(B_{2}\right)$ & 12.7 & yes & B0.5 I & SFXT? \\
\hline XTE J1739-302 & J17391155-3020380 & $0596-0585865$ & +0.07 & 7.43 & 17.3 & 13.2 & yes & $\mathrm{O} 8.5 \mathrm{Iab}(\mathrm{f})$ & SFXT \\
\hline IGR J17544-2619 & J17542527-2619526 & $0636-0620933$ & -0.02 & 8.02 & 14.5 & 11.3 & yes & O9 Ib & SFXT \\
\hline SAX J1818.6-1703 & $\mathrm{J} 18183790-1702479$ & 0729-0750578 & +0.09 & 7.85 & $\ldots$ & 16.8 & likely & O9-B1 I & SFXT \\
\hline IGR J11305-6256 & J11310691-6256489 & $0270-0309619$ & -0.12 & 8.01 & 8.1 & 8.2 & yes & B0.5 IIIe & HD 100199 \\
\hline IGR J16207-5129 & $\mathrm{J} 16204627-5130060$ & $0384-0560875$ & -0.01 & 9.13 & $\ldots$ & 15.2 & yes & B0 I & SGXB \\
\hline IGR J16283-4843 & $\mathrm{J} 16280983-4838590$ & $0413-0504855$ & -0.30 & 12.01 & $\ldots$ & 14.0 & no & K & $\ldots$ \\
\hline IGR J16320-4751 & $\mathrm{J} 16320175-4752289$ & $\cdots$ & $\cdots$ & 10.99 & $\cdots$ & $\cdots$ & likely & $\ldots$ & $\cdots$ \\
\hline & $\mathrm{J} 16320215-4752322$ & $0421-0625270$ & +0.18 & 10.82 & $17.3\left(B_{2}\right)$ & 14.6 & no & K & $\ldots$ \\
\hline AX J1700.2-4220 & $\mathrm{J} 17002524-4219003$ & $0476-0587932$ & -0.26 & 6.73 & 9.5 & 8.8 & perhaps & B0.5 IVe & HD 153295 \\
\hline IGR J17091-3624 & $\mathrm{J} 17090199-3623260$ & $0536-0466988$ & +0.00 & 11.7 & $\ldots$ & 14.46 & likely & F8 V & Radio source \\
\hline AX J1820.5-1434 & $\mathrm{J} 18203114-1434193$ & $0754-0489829$ & +0.06 & 10.98 & $16.5\left(B_{2}\right)$ & $14.7\left(R_{2}\right)$ & perhaps & B & not $\mathrm{Be}$ \\
\hline IGR J18406-0539 & $\mathrm{J} 18404689-0540502$ & $0843-0409384$ & -0.05 & 8.613 & 12.7 & 11.4 & unlikely & $\mathrm{Mn}-\mathrm{Hg}$ & SS 406 \\
\hline IGR J16393-4641 & J16390535-4642137 & $\ldots$ & +0.41 & 12.78 & $\ldots$ & $\ldots$ & candidate & $\ldots$ & $\ldots$ \\
\hline IGR J16418-4532 & $\mathrm{J} 16415078-4532253$ & $\ldots$ & +0.16 & 11.48 & $\ldots$ & $\ldots$ & candidate & $\ldots$ & $\ldots$ \\
\hline IGR J16479-4514 & $\mathrm{J} 16480656-4512068$ & $0447-0531332$ & +0.38 & 9.80 & .. & 18.36 & candidate & $\ldots$ & $\ldots$ \\
\hline IGR J17252-3616 & $\mathrm{J} 17251139-3616575$ & $\ldots$ & +0.47 & 10.67 & $\ldots$ & $\ldots$ & candidate & $\ldots$ & $\ldots$ \\
\hline
\end{tabular}

3. Our method reveals a B-type star inside the error circle for the X-ray pulsar AX J1820.5-1434, but our spectra are not good enough to decide if this is an early B-type star (and hence a potential candidate). $\mathrm{H} \alpha$ seems to be in absorption.

4. For HD 100199, identified with IGR J11305-6256, we confirm the spectral type B0 IIIe.

5. We fail to find any suitable counterpart within the $A S C A$ error circle for AX J1700.2-4220. For the suggested counterpart, HD 153295, which is inside the INTEGRAL error circle, but not the ASCA circle, we find a spectral type B0.5 IVe and a distance compatible with membership in the Sco OB1 association.

6. In the error circle for the likely black hole transient IGR J17091-3624, a variable radio source has been detected. The star likely associated with this radio source is $\sim \mathrm{F} 8 \mathrm{~V}$ and must be much closer than the Galactic Bulge.

7. We rule out the association of SS 406 with IGR J18406-0539, as this object is a late B chemically peculiar star, and perhaps a binary consisting of two unevolved stars.

Acknowledgements. We would like to thank Andrew J. Norton for helpful comments on the manuscript and the referee, Doug Gies, for many valuable suggestions that improved notably the quality of the paper. IN is a researcher of the programme Ramón y Cajal, funded by the Spanish Ministerio de Educación y Ciencia and the University of Alicante, with partial support from the Generalitat Valenciana and the European Regional Development Fund (ERDF/FEDER). This research is partially supported by the MEC under grant AYA2005-00095. During part of this work, I.N. was a visiting fellow at the Open University, whose kind hospitality is warmly acknowledged. This visit was funded by the MEC under grant PR2006-0310.

This research has made use of the Simbad data base, operated at CDS, Strasbourg (France). This publication makes use of data products from the Two Micron All Sky Survey, which is a joint project of the University of Massachusetts and the Infrared Processing and Analysis Center/California Institute of Technology, funded by the National Aeronautics and Space Administration and the National Science Foundation. It also makes use of DENIS, the result of a joint effort involving human and financial contributions of several Institutes mostly located in Europe. It has been supported financially mainly by the French Institut National des Sciences de l'Univers, CNRS, and French Education Ministry, the European
Southern Observatory, the State of Baden-Wuerttemberg, and the European Commission under networks of the SCIENCE and Human Capital and Mobility programs, the Landessternwarte, Heidelberg and Institut d'Astrophysique de Paris. This paper makes use of data from the UVES Paranal Observatory Project (ESO 266.D-5655).

Partially based on observations made with the Nordic Optical Telescope, operated on the island of La Palma jointly by Denmark, Finland, Iceland, Norway, and Sweden, in the Spanish Observatorio del Roque de los Muchachos of the Instituto de Astrofisica de Canarias. Part of the data presented here have been taken using ALFOSC, which is owned by the Instituto de Astrofísica de Andalucía (IAA) and operated at the Nordic Optical Telescope under agreement between IAA and the NBIfAFG of the Astronomical Observatory of Copenhagen.

\section{References}

Bagnulo, S., Jehin, E., \& Ledoux, C. 2003, Messenger, 114, 10 Beckmann, V., Kennea, J. A., Markwardt, C., et al. 2005, ApJ, 631, 506 Bessell, M. S., \& Brett, J. M. 1988, PASP, 100, 1134 Bird, A. J., Barlow, E. J., Bassani, L., et al. 2004, ApJL, 607, 33 Bodaghee, A., Walter, R., Zurita Heras, J. A., et al. 2006, A\&A, 447, 1027 Capitanio, F., Bazzano, A., Ubertini, P., et al. 2006, ApJ, 643, 376 Caron, G., Moffat, A. F. J., St-Louis, N., et al. 2003, AJ, 126, 1415

Clark, J. S., Negueruela, I., Crowther, P. A., \& Goodwin, S. P. 2005, A\&A, 434, 949

Comerón, F., \& Pasquali, A. 2005, A\&A, 430, 541

Corbet, R., Barbier, L., Barthelmy, S., et al. 2005, ATel, 649

Dachs, J., Kiehling, R., \& Engels, D. 1988, A\&A, 194, 167

Ducati, J. R., Bevilacqua, C. M., Rembold, S. B., \& Ribeiro, D. 2001, ApJ, 558, 309

Draper, P. W., Taylor, M., \& Allan, A. 2000, Starlink User Note 139.12, R.A.L.

Filippova, E. V., Tsygankov, S. S., Lutovinov, A. A., \& Sunyaev, R. A. 2005, Astron. Lett., 31, 729

Filliatre, P., \& Chaty, S. 2004, ApJ, 616, 469

Fitzpatrick, E. L. 1999, PASP, 111, 63

Garrison, R. F., Hiltner, W. A., Schild, R. E., et al. 1977, ApJS, 35, 111 Grimm, H.-J., Gilfanov, M., \& Sunyaev, R. 2003, MNRAS, 339, 793

Howarth, I., Murray, J., Mills, D., \& Berry, D. S. 1998, Starlink User Note 50.21, R.A.L.

Humphreys, R. M., \& McElroy, D. B. 1984, ApJ, 284, 565

Indebetouw, R., Mathis, J. S., Babler, B. L., et al. 2005, ApJ, 619, 931

Kaper, L., van der Meer, A., \& Tijani, A. H. 2004, RMxAC, 21, 128

Kinugasa, K., Torii, K., Hashimoto, Y., et al. 1998, ApJ, 495, 435

Koornneef, J. 1985, A\&A, 128, 84 
Kuulkers, E., Lutovinov, A., Parmar, A., et al. 2003, ATel, 149

Lutovinov, A., Rodriguez, J., Revnivtsev, M., \& Shtykovskiy, P. 2005a, A\&A, 433, L41

Lutovinov, A., Revnivtsev, M., Molkov, S., \& Sunyaev, R. 2005b, A\&A, 430, 997

Masetti, N., Pretorius, M. L., Palazzi, E., et al. 2006a, A\&A, 449, 1139

Masetti, N., Morelli, L., Palazzi, E., et al. 2006b, A\&A, 459, 21

Masetti, N., Mason, E., Bassani, L., et al. 2006c, A\&A, 448, 547

Mikami, T., \& Heck, A. 1982, PASJ, 34, 529

Negueruela, I., \& Smith, D. M. 2006, ATel, 831

Negueruela, I., Smith, D. M., \& Chaty, S. 2005, ATel, 429

Negueruela, I., Smith, D. M., Harrison, T. E., \& Torrejón, J. M. 2006a, ApJ, 638, 982

Negueruela, I., Smith, D. M., Reig, P., et al. 2006b, ESA SP-604, 165

Palla, F., \& Stahler, S. W. 1999, ApJ, 525, 772

Pandey, M., Manchanda, R. K., Rao, A. P., et al. 2006, A\&A, 446, 471

Pellizza, L. J., Chaty, S., \& Negueruela, I. 2006, A\&A, 455, 653

Pfahl, E., Rappaport, S., \& Podsiadlowski, P. 2002, ApJ, 571, L37

Produit, N., Ballet, J., \& Mowlavi, N. 2004, ATel, 278
Raboud, D. Cramer, N., \& Bernasconi, P. A. 1997, A\&A, 325, 167

Reig, P., Negueruela, I., Papamastorakis, G., et al. 2005, ApJ, 440, 637

Ribó, M., Negueruela, I., Blay, P., et al. 2006, A\&A, 449, 687

Rieke, G. H., \& Lebofsky, M. J. 1985, ApJ, 288, 618

Rodriguez, J., Bodaghee, A., Kaaret, P., et al. 2006, MNRAS, 366, 274

Rupen, M. P., Mioduszewski, A. J., \& Dhawan, V. 2003, ATel, 152

Schild, R. E., Neugebauer, G., \& Westphal, J. A. 1971, AJ, 76, 237

Sguera, V., Barlow, E. J., Bird, A. J., et al. 2005, A\&A, 444, 221

Skrutskie, M. F., Cutri, R. M., \& Stiening, R. 2006, AJ, 131, 1163

Shortridge, K., Meyerdicks, H., Currie, M., et al. 1997, Starlink User Note 86.15, R.A.L.

Sidoli, L., Paizis, A., \& Mereghetti, S. 2006, A\&A, 450, L9

Smith, D. M., Heindl, W. A., Markwardt, C. A., et al. 2006, ApJ, 638, 974

Steeghs, D., Torres, M. A. P., Jonker, P. G., et al. 2005, ATel, 478

Thé, P. S., de Winter, D., \& Perez, M. R. 1994, A\&AS, 104, 315

Tomsick, J. A., Chaty, S., Rodriguez, J., et al. 2006, ApJ, 647, 1309

Walter, R., Zurita Heras, J. A., Bassani, L., et al. 2006, A\&A, 453, 133

Wegner, W. 1994, MNRAS, 270, 229

Zurita Heras, J. A., de Cesare, G., Walter, R., et al. 2006, A\&A, 448, 261 